\title{
Observations of coarsening of air voids in a polymer-highly-soluble crystalline matrix during dissolution
}

\author{
Eleni Karakosta, Paul M. Jenneson, ${ }^{*}$ Richard P. Sear, and Peter J. McDonald \\ Department of Physics, School of Electronics and Physical Sciences, University of Surrey, Guildford, \\ Surrey, GU2 7XH, United Kingdom
}

(Received 24 February 2006; published 17 July 2006)

\begin{abstract}
A combination of magnetic resonance imaging and $\mathrm{x}$-ray microcomputed tomography has been used to visualize the development of the internal micro-structure within compressed tablets made from a combination of insoluble particles (Eudragit, a polymer) and soluble particles (diltiazem hydrochloride, a drug), during dissolution in water. Air voids in the tablet are seen to coarsen. The size distribution of the air voids is well fitted by a log-normal distribution with a mean size that grows linearly with time. There is evidence for both diffusion of voids and sudden collapse of individual voids, presumably as they coalesce. The behavior of the voids is studied and compared with models of coarsening; the implications for tablet dissolution are considered.
\end{abstract}

DOI: 10.1103/PhysRevE.74.011504

PACS number(s): 64.75. $+\mathrm{g}$, 76.60.Pc, 47.56.+r

\section{INTRODUCTION}

Soluble tablets are used as delivery agents across a wide spectrum of technology sectors, from agriculture to medicine. They are made by compressing powders, and they inevitably contain some air voids between the particles. An obvious question to ask is, what happens to these air voids as the tablet dissolves? We address this question quantitatively for a model tablet system comprising a compressed mixture of insoluble polymer and soluble drug particles. We do so by using x-ray computed microtomography (CT) and magnetic resonance microimaging (MRI), combined with a simple theoretical analysis. The X-ray CT method in particular allows us to noninvasively obtain the probability distribution function of the void size in a single tablet at a number of different times throughout the dissolution process. We find that the voids coarsen, i.e., grow, as the time since the tablet was immersed in water increases. Our analysis is similar to that developed for other systems that undergo coarsening, such as the domains in a phase-separating mixture $[1,2]$ and in the droplets of a coarsening emulsion $[3,4]$.

Motivation for studying the dissolution of a soluble component mixed as particles with an insoluble component is provided by tablet use in the controlled release of drugs, in agricultural products such as pesticides, and in a range of cleaning products such as detergents. Although simple models of these materials have been studied [5], the successful design of polymer matrix controlled release tablets remains something of a black art, constrained by a restricted understanding of the mechanisms of dissolution. With better experimental data and hence understanding would come the ability to model the behavior of current delivery systems and confidently predict the behavior of future systems.

Tablets made of compressed powders contain structure over length scales from angstroms, the interfaces between particles and the surrounding air or water, to many microme-

\footnotetext{
*Corresponding author. FAX: +44 1483 686781. Email address: p.jenneson@surrey.ac.uk
}

ters, the air voids and large particles, to millimeters, the size of individual tablets. This makes their behavior potentially complex and therefore hard to understand and hard to predict. For example, the rate of dissolution of the soluble component potentially depends on the dissolution rate at the interface between water and its crystalline particles; on its overall solubility and concentration-dependent diffusion constant; on any potential swelling of the insoluble matrix; as well as on structure on larger length scales, for example the porosity, which may well evolve with time. Thus a comprehensive study will require applying a range of techniques to probe a number of phenomena over a range of length scales. Here we use x-ray computed microtomography [6] and magnetic resonance microimaging [7] to probe the larger length scales. Spectroscopic techniques can also be used, e.g., Fourier transform infrared (FTIR) [8] and Raman spectroscopy [9].

The fact that the systems are wet, soft, and evolving with time hampers direct measurement. X-ray CT and MRI have the ability to provide internal images of soft materials under study and to visualize the sample in three dimensions. Both $\mathrm{x}$-ray CT and MRI are nondestructive and noninvasive. MRI is firmly established as a valuable tool for analyzing liquid transport in polymers and polymer dissolution. It can be used to follow water ingress into polymer matrices $[10,11]$ the polymer swelling and dissolution [12], and the release of some soluble components $[13,14]$. Image contrast is strongly weighted by molecular motion, providing a means to visualizing different components. Maps of the water distribution and, in appropriate cases, the dissolved component also, can be obtained and used to test theoretical models. X-ray CT is an imaging technique that can also provide cross-sectional images in different planes through a sample and has recently been advanced in the area of drug development. Sinka et al. [6] measured the material density distribution in pharmaceutical tablets noninvasively by x-ray CT. Rather than mobility, image contrast is dominated by electron density. CT is therefore complementary to MRI.

In the next section we briefly describe our material: an insoluble, and also nonswelling, polymer matrix containing a 
soluble drug that we expose to water. Section III describes our techniques and Sec. IV presents our results, while Sec. V analyzes these in the light of competing models for coarsening. It is shown that the growth of these air voids is consistent with a stochastic model of ripening involving discrete coalescence events. The final section is a conclusion.

\section{MATERIALS}

The polymer used in this study is Eudragit RSPO (Degussa Pharma Polymers, Darmstadt, Germany). It is commonly used in film coating of tablets, granules, and other small particles and also in matrix formulation. It is a copolymer of acrylic and methacrylic acid ester with low content of quaternary ammonium groups. The ammonium groups are present as salts and make the polymer permeable. Eudragit is generally considered to be nonswelling in water [15] although there is some evidence that it does swell slightly in digestive tract fluids $[16,17]$. We find that a tablet made from only Eudragit swells by $\approx 4 \%$ and we do not observe the air voids found in the mixed Eudragit-soluble component tablets. The polymer particle size was graded $45-100 \mu \mathrm{m}$. The soluble drug is diltiazem hydrochloride (Spectrum Chemical and Laboratory Products, Gardena, California, USA), a calcium ion cellular influx inhibitor. Diltiazem hydrochloride is a solid crystalline powder, highly soluble in water. The drug particle size distribution was also graded 45-100 $\mu \mathrm{m}$.

\section{METHODS}

\section{A. ${ }^{1} \mathrm{H}$ imaging}

Imaging experiments were carried out using a ${ }^{1} \mathrm{H}$ $400 \mathrm{MHz}$ Chemagnetics Infinity Spectrometer (Varian Limited, Oxford, UK) coupled to a Magnex $9.4 \mathrm{~T}$ (89 mm widebore) superconducting magnet (Magnex Scientific, Oxon, UK). The spectrometer was equipped with a ${ }^{1} \mathrm{H}$ microscopy probe (IBMT, Fraunhofer-Institute für Biomedizinische Technik IBMT, St. Ingebert, Germany) and imaging gradients (Resonance Research Instruments, Austin, Texas, USA).

For the MRI measurements reported here, tablets composed of a mixture of polymer and drug were prepared on a pure polymer tablet base. This provided greater structural integrity during the dissolution of tablets with a high drug fraction. To make such samples, a base layer of Eudragit powder was first lightly pressed in an instrumented singlepunch tablet pressing machine (IP Technical Services, Richmond, UK) equipped with $9 \mathrm{~mm}$ flat-faced punches. A mixture of Eudragit and drug in the volume ratio $45 \%$ polymer to $55 \%$ drug was then added above this layer and the whole sample fully compressed at a pressure of $0.30 \mathrm{GPa}$. The manufactured sample matrix was therefore $9 \mathrm{~mm}$ in diameter. The height of the sample was $3.0 \pm 0.2 \mathrm{~mm}$. The samples were placed in $9 \mathrm{~mm}$ internal diameter NMR tubes, supported from below on a thin polytetrafluoroethylene (PTFE) collar. The collar enabled both sides of the tablet to be exposed to a volume of water large compared to the sample volume. However, water substantially ingressed the mixed layer only from above. The water was lightly doped (3.7 $\left.\times 10^{-4} M\right)$ with copper sulfate $\left(\mathrm{CuSO}_{4}\right.$, Fisher Scientific UK
Ltd, Loughborough, UK) in order to reduce the ${ }^{1} \mathrm{H}$ nuclear spin-lattice relaxation time. Measurements were also performed using a phosphate buffer solution (without $\mathrm{CuSO}_{4}$ ) to modify the $p \mathrm{H}$. Although these measurements took longer to record for equivalent signal-to-noise ratio, due to the increased spin-lattice relaxation time, the results were not substantially different from those reported here. The MR images were obtained using a standard spin-echo imaging sequence [18] with an echo time of $4 \mathrm{~ms}$. Coronal slices at the center of the tablet (that is, slices containing the longitudinal symmetry axis of the sample and one diameter) with thickness of $1 \mathrm{~mm}$ were imaged. The image field of view was 15 $\times 24 \mathrm{~mm}^{2}$ with an in-plane pixel resolution of 29 $\times 188 \mu \mathrm{m}^{2}$ (the better resolution being in the longitudinal direction). The acquisition time of each image was $34 \mathrm{~min}$.

\section{B. X-ray computed microtomography}

The microstructure of the sample matrix was observed using a bench-top third-generation cone-beam x-ray computed microtomography $(\mu \mathrm{CT})$ scanner [19]. The x-ray detector used was a Hamamatsu C7942 flat-panel complementary metal oxide semiconductor array and the x-ray source used was a Hamamatsu L6731-01 microfocus x-ray tube with a focal spot size of $5 \mu \mathrm{m}$ operated at a peak energy of $55 \mathrm{kV}$ and a filament current of $0.1 \mathrm{~mA}$. The distance between the source and the sample was $50 \mathrm{~mm}$ whereas the distance between the sample and the x-ray detector was $450 \mathrm{~mm}$.

The measurements were conducted on samples manufactured in an identical way to those used for MRI except that the polymer and drug fractions were $75 \%$ and $25 \%$, respectively; the sample diameter and height were both $3 \mathrm{~mm}$; and the compression pressure was $0.14 \mathrm{GPa}$. The smaller drug fraction was used for two reasons. First it slowed the dissolution to a rate comparable to that of image data acquisition. Second, it improved the structural integrity of the tablets during dissolution. The samples were exposed to distilled water. $450 \mathrm{x}$-ray radiograph projections were acquired while rotating the sample through $360 \mathrm{deg}$. The detector digitized the projection data into $512 \times 512$ pixels with a pixel depth of 14 bits. The total scan time was $40 \mathrm{~min}$, comparable to that for MRI. The x-ray projections contain superimposed images of the sample which were recovered into a threedimensional data set using a Feldkamp cone-beam back projection routine [20]. The magnification of the source-sample and sample-detector distances resulted in a voxel dimension of $10 \times 10 \times 10 \mu \mathrm{m}^{3}$ over a field of view of $5.12 \mathrm{~mm}$ in each direction.

\section{RESULTS}

\section{A. ${ }^{1}$ H MRI imaging}

Figure 1 shows a time series of ${ }^{1} \mathrm{H}$ MRI two-dimensional images of the polymer-drug matrix sample exposed to water. The images each took 34 min to acquire. The contrast results almost exclusively from differences in molecular mobility of water, polymer, and drug. The lighter upper and lower regions of the images are the water. The apparently fading top 
(a)

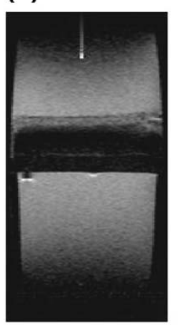

(e)

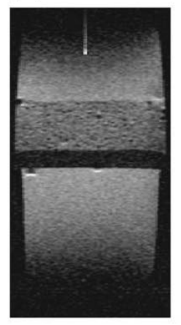

(b)

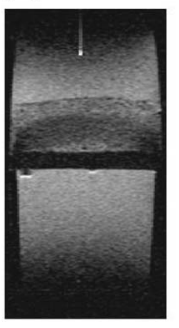

(f)

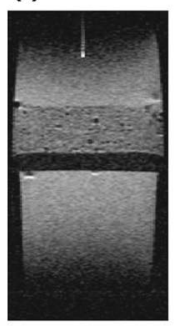

(c)

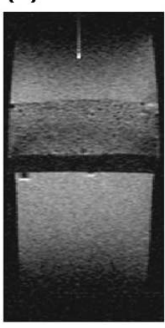

(g)

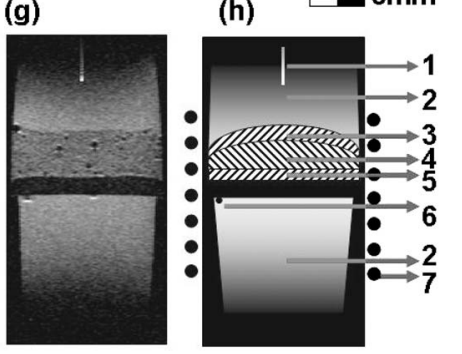

(d)
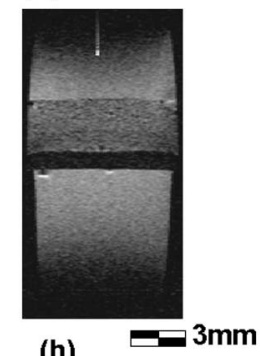

FIG. 1. ${ }^{1} \mathrm{H}$ images of a sample matrix exposed to water at room temperature. Central coronal slice of $1 \mathrm{~mm}$ in thickness and inplane resolution of $29 \mu \mathrm{m}$ coronally $\times 188 \mu \mathrm{m}$ axially. The exposure times are (a) 34, (b) 68, (c) 137, (d) 239, (e) 478, (f) 956, and (g) 1912 min. (h) Annotated diagram where (1) imaging artifact, (2) bulk water, (3) water in tablet and accompanying drug dissolution, (4) capillary water in tablet open porosity, (5) supporting Eudragit layer, (6) air bubble, (7) PTFE support, and (8) sensor coils.

and bottom shows where the tube extends beyond the sensitive region of the scanner defined by the sensor coil. The dark horizontal strip in the middle is the polymer base to the tablet, which is scarcely affected by the water. However, careful numerical analysis of the image intensity suggests it rapidly takes up circa $4 \%$ water. Above this is the tablet. In the first image, it is largely uninvaded by water. Again, however, detailed analysis of the signal intensity in this (and other images recorded more quickly at lower resolution) coupled with gravimetric analysis suggests that water rapidly absorbs into the open porosity of the tablet. When drug is present, this corresponds to circa $2 \%$ of the total volume, less than with pure polymer. In the subsequent time series, a sharp, clear water diffusion front is seen to invade the tablet. It is noted that the front advances as the square root of time. More importantly, as far as this work is concerned, a series of air voids, visualized as dark spots, are seen to form and grow. Curiously, these air voids develop both behind and in front of the primary diffusion front. Corroborative evidence that these dark spots are indeed air voids comes from the fact that they display the classic artifact of a bright streak in the direction of the so-called MRI "read" or "frequency encode" gradient. The streaks are caused by the significant difference in magnetic susceptibility between the void space (air) and the matrix (polymer-drug-water) [21].

\section{B. X-ray $\mu \mathrm{CT}$}

The MRI results show interesting changes in the microstructure of the sample. X-ray $\mu \mathrm{CT}$ was employed to probe it on a finer length scale. The reconstructed X-ray CT data are

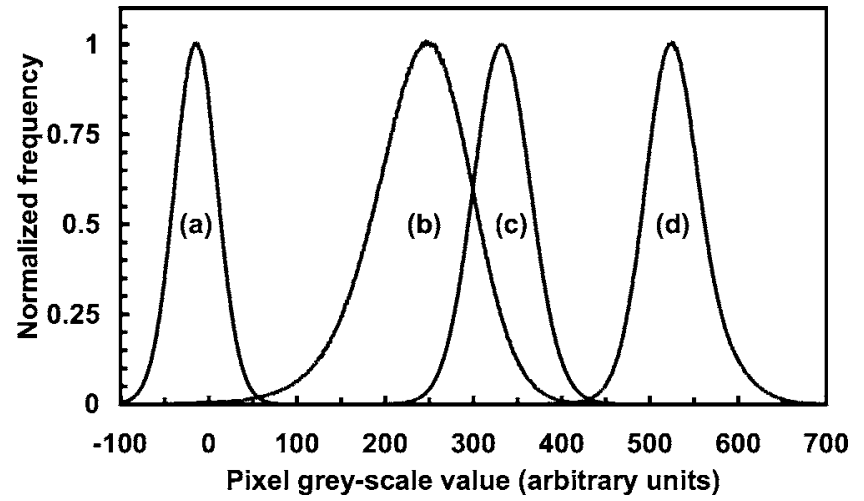

FIG. 2. Histogram of the gray-scale values of the reconstructed $\mathrm{x}$-ray $\mu \mathrm{CT}$ data for (a) air, (b) polymer, (c) water, and (d) drug.

essentially a set of consistent but uncalibrated gray-scale values. By separately scanning samples of pressed polymer, pressed drug, water, and air under the same acquisition parameters the gray-scale values can be assigned to either polymer, drug, air or water (see Fig. 2). In a dry system, polymer and drug can be easily distinguished. In a wet system, the water dramatically complicates the analysis as it has a very similar electron density and composition to the polymer. The gray-scale values overlap. Moreover, in mixed systems, partial filling of voxels by more than one component leads to intermediate gray-scale values. From the image analysis viewpoint, this is a serious complication which hinders quantitative analysis. Notwithstanding this, air-filled voxels are easily distinguished from the matrix.

Figure 3 shows the central coronal slices extracted from a three-dimensional data set of a tablet exposed to water. In Fig. 3(a), which is an image of the dry system, the tablet is clearly seen as the dappled region. The dark space above and to the sides is air space into which the water is subsequently placed [Figs. 3(b)-3(h)]. The uniformly gray stripes to the side are a machined polymer tube designed to hold the (a)

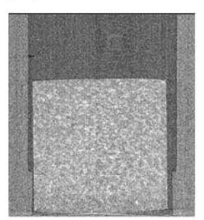

(e)

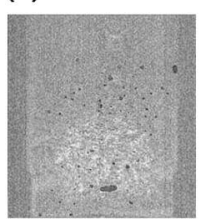

(b)

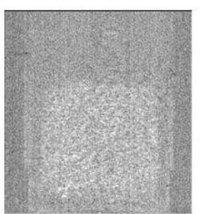

(f)

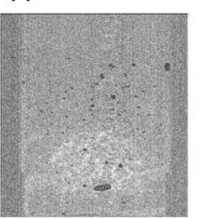

(c)

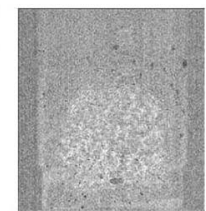

(g)

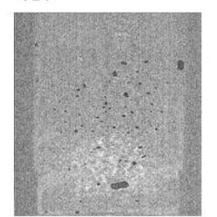

(d)
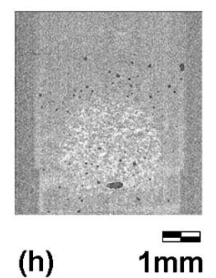

(h)

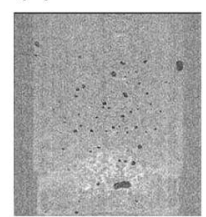

FIG. 3. The central coronal slice extracted from a threedimensional x-ray $\mu \mathrm{CT}$ data set. Slice (a) is a dry matrix. The tablet is the speckled rectangle, lower center. Air appears dark and the side gray stripes are a polymer tube supporting the sample. (b)-(h) show the same slice exposed to water for the following times: 45, $90,135,180,225,270$, and $315 \mathrm{~min}$. Water appears a light gray, comparable to the tablet. 


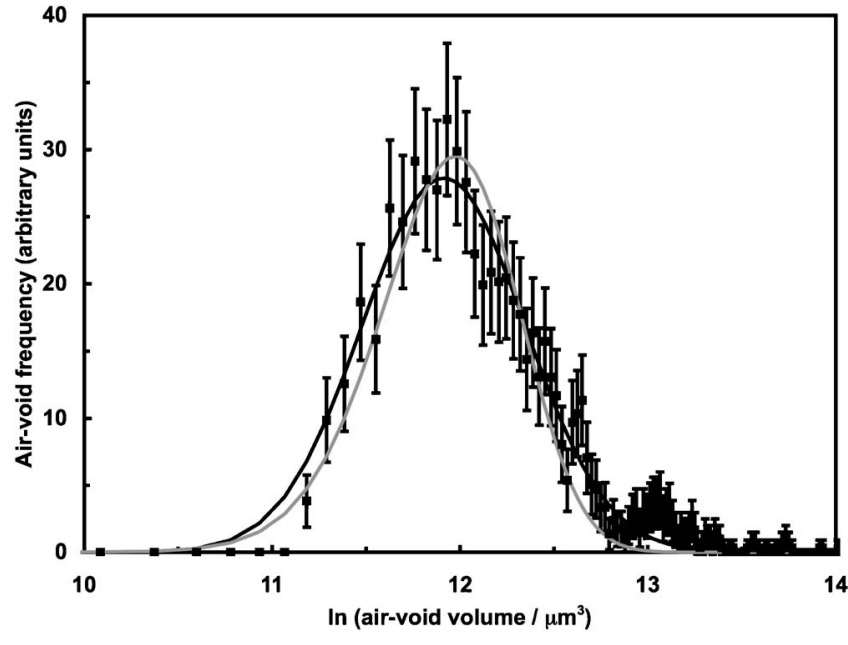

FIG. 4. The histogram of the natural logarithm of the frequency of air voids versus air void volume $\left(\mu \mathrm{m}^{3}\right)$ for a sample exposed to water for $270 \mathrm{~min}$. The squares are the data points and error bars are indicated. The black and gray curves are log-normal and Schulz distribution fits to the data.

sample. Figures 3(b)-3(h) show a time series of images of water ingressing into the tablet. As with the MRI results, it is seen that after adding water, the tablet quickly divides into two parts: that in which water has clearly invaded and the drug component has been dissolved (an outer region) and that where it has invaded to a much less extent (the inner region). Ingress into the inner region results from capillary uptake into the open porosity of the tablet. The two regions are again separated by a sharp front. The former grows at the expense of the latter. Again air voids are seen to form and grow with time. As with MRI, the voids are seen in both the inner and outer regions of the tablet.

The air-void size distribution $p(V)$ was extracted from the three-dimensional $\mathrm{x}$-ray $\mu \mathrm{CT}$ data. This was done using the gray-scale values, shown in Fig. 2, to apply a binary threshold to reveal just the air voids. Face connected pixels were discretely assigned to be part of a single void and so an air-void volume size distribution was determined. The void size distribution is obtained for the whole tablet, notwithstanding the fact that the ingressing solvent divides the tablet into two regions as discussed above. A typical distribution is shown in Fig. 4, this example extracted from the data set at $270 \mathrm{~min}$. It is found that the void size distribution is well characterized by a log-normal size distribution

$$
p(V)=\frac{1}{(2 \pi)^{1 / 2} \sigma V} \exp \left[-\frac{1}{2}\left(\frac{\ln (V)-\overline{\ln (V)}}{\sigma}\right)^{2}\right]
$$

where $\sigma$ is the standard deviation of $\ln (V)$ centered on the mean value $\overline{\ln (V)}$. The solid black curve of Fig. 4 is a fit of this function to the data.

The true mean air-void volume $\bar{V}$ is simply related to $\overline{\ln (V)}$ by

$$
\bar{V}=\exp \left(\frac{2 \overline{\ln (V)}+\sigma^{2}}{2}\right)
$$

and is plotted as a function of time in Fig. 5. It is seen to

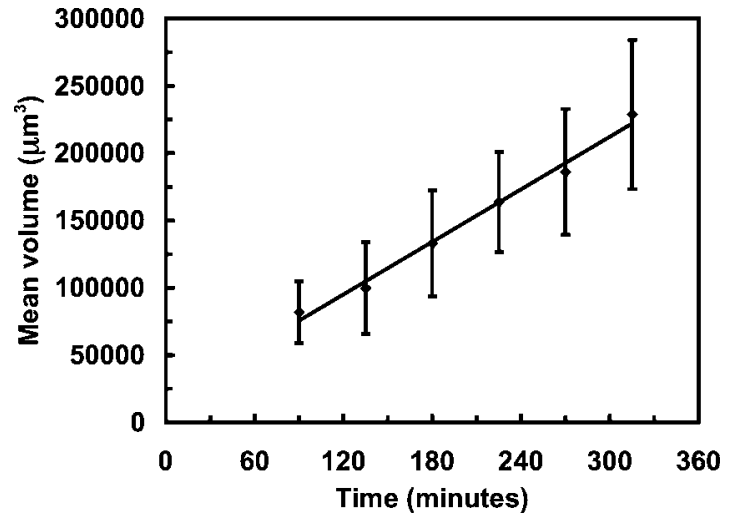

FIG. 5. The increase in the mean air-void volume in $\mu \mathrm{m}^{3}$ with respect to time. The diamonds are the means and the bars represent the standard deviation of the size distribution above and below the mean.

increase linearly with time. In order to assess the mechanism for this growth, the four largest air voids, by volume, were digitally extracted from the central $500 \times 500 \times 500 \mu \mathrm{m}^{3}$ region of the tablet. Figure 6 shows a three-dimensional volume rendering of the four largest air voids at 180, 225, 270, and $315 \mathrm{~min}$. The total accumulated volume of these four air voids is $1.16 \times 10^{6}, 1.51 \times 10^{6}, 1.32 \times 10^{6}$, and 1.10 $\times 10^{6} \mu \mathrm{m}^{3}$, respectively. Figure 6(e) shows the movement of the centers of mass of the four voids. The individual void sizes and movement are presented in Tables I and II, respectively, together with measurement errors.

The data of Fig. 6 and Tables I and II are noisy but it is notable that over the time period there is no trend in the volume of three of the voids, 1,2 , and 3 , whereas for the void number 4 , there is no detectable trend over the first three times but between the third and fourth times, its volume decreases dramatically. This volume may be redistributing into the nearby void number 3 but our data are too noisy to prove or disprove this. Note that although void 4 is close to other voids it does not appear to be in contact with them. Thus coalescence may not involve voids diffusing into contact, as emulsion droplets do, but instead only approaching closely enough that the air can cross a narrow region of porous matrix between them. However, the actual mechanism of air transport is on length scales smaller than those we can observe with the two techniques employed here.

The four chosen voids are not typical: they are among the largest in the sample. They are chosen as their large size (a)

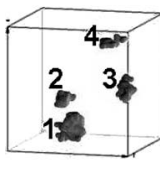

(b)

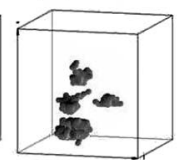

(c)

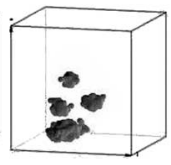

(d)

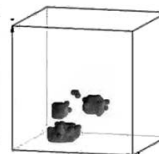

(e)

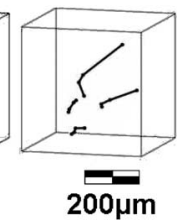

FIG. 6. Three-dimensional volume renderings of the four largest air voids at (a) $180 \mathrm{~min}$ (total air volume $0.00116 \mathrm{~mm}^{3}$ ), (b) $225 \mathrm{~min}$ (total air volume $0.00151 \mathrm{~mm}^{3}$ ), (c) $270 \mathrm{~min}$ (total air volume $0.00132 \mathrm{~mm}^{3}$ ), and (d) $315 \mathrm{~min}$ (total air volume $0.00110 \mathrm{~mm}^{3}$ ). (e) shows the movement of the centers of mass for the four air voids. 
TABLE I. Physical measurements for the four largest air voids in the central $500 \times 500 \times 500 \mu \mathrm{m}^{3}$ region of the tablet. The error bars of $\pm 25 \%$ are estimates based on an assumption that the volume of one void, chosen to be void 1 , is not changing with time and that the fluctuations in its measured volume are due to imaging errors.

\begin{tabular}{ccccc}
\hline \hline & \multicolumn{4}{c}{ Air-void size $\left(10^{5} \mu \mathrm{m}^{3}\right)$} \\
\cline { 2 - 5 }$(\min )$ & 1 & 2 & 3 & 4 \\
\hline 180 & $5.4 \pm 1.3$ & $2.1 \pm 0.5$ & $2.4 \pm 0.6$ & $1.8 \pm 0.4$ \\
225 & $7.3 \pm 1.9$ & $3.4 \pm 0.9$ & $1.7 \pm 0.4$ & $2.6 \pm 0.6$ \\
270 & $6.7 \pm 1.6$ & $2.3 \pm 0.6$ & $2.6 \pm 0.7$ & $1.7 \pm 0.4$ \\
315 & $5.5 \pm 1.4$ & $2.1 \pm 0.5$ & $3.3 \pm 0.8$ & $0.19 \pm 0.05$ \\
\hline \hline
\end{tabular}

makes them easier to render. Attempts to repeat the exercise with smaller voids encounters two problems: first the measurement errors become larger since the void surface-tovolume ratio is increased; second, the voids are more numerous and move more quickly so that it is harder to unambiguously track the motion of specific voids given the frame rate of the available data.

\section{ANALYSIS AND THEORETICAL MODEL}

The experimental results show three main features: (1) the probability distribution function of air voids is well fitted by a log-normal distribution function, (2) the mean volume increases linearly with time, and (3) there is evidence the volume of a void can suddenly collapse, presumably as its volume is distributed to adjacent voids.

We assume that the ultimate state of our system would be a single air void, which might escape, plus a solid matrix without voids. The process by which initially small domains, here our air voids, start off small, but coarsen over time to become larger and larger has been widely studied. It occurs in systems undergoing phase separation, and the droplets in an emulsion also tend to coarsen. Thus, attempts to understand the coarsening of droplets, particles, or voids have a long history. Two main mechanisms have been studied extensively: coarsening via diffusion from small to large droplets driven by chemical potential gradients, called Ostwald ripening, and coarsening by the diffusion of droplets into contact followed by their coalescence.

TABLE II. The relative movements of the four voids in Table I. These are the displacements from 180 to $225 \mathrm{~min}$, from 225 to $270 \mathrm{~min}$, etc. The error bars are the actual image resolution $10 \mu \mathrm{m}$.

\begin{tabular}{lcccc}
\hline \hline & \multicolumn{4}{c}{ Movement $(\mu \mathrm{m})$} \\
\cline { 2 - 5 } \begin{tabular}{c} 
Time $(\min )$ \\
\cline { 2 - 5 }
\end{tabular} & 1 & 2 & 3 & 4 \\
\hline 180 & & & & \\
225 & $43 \pm 10$ & $43 \pm 10$ & $43 \pm 10$ & $43 \pm 10$ \\
270 & $30 \pm 10$ & $30 \pm 10$ & $30 \pm 10$ & $30 \pm 10$ \\
315 & $13 \pm 10$ & $13 \pm 10$ & $13 \pm 10$ & $13 \pm 10$ \\
\hline \hline
\end{tabular}

The theory for coarsening via diffusion of molecules down chemical potential gradients is well developed. It was initially introduced by Lifshitz and Slyozov [22] and Wagner [23] (LSW) and much work has been done since [24,25]. Here, the smaller droplets have large surface-to-volume ratios and so the surface tension contributes a larger amount to the chemical potential in these smaller droplets than in the larger droplets. So there is a chemical potential gradient from small to large droplets down which molecules diffuse, thus causing the large droplets to grow at the expense of the smaller droplets. Dickinson et al. [26] studied the Ostwald ripening kinetics in $n$-alkane oil-in-water emulsions stabilized by sodium caseinate to monitor the time-dependent changes in the average droplet diameter and droplet size distribution. They found a linear increase of droplet size with time stored. In Ostwald ripening, the growth (shrinkage) of the large (small) droplets is continuous and, as LSW showed, the distribution of droplet sizes at late times is rather narrow with a rather sharp cutoff at large sizes. Our distribution of void volumes is well fitted by a log-normal distribution which is rather different from the LSW distribution; in particular, there is no cutoff at large sizes. However, LSW do predict a linear growth of the mean void volume with time, which is what we observe (see Fig. 5). Finally, LSW predict that the four large droplets shown in Fig. 6 should grow smoothly at the expense of the smaller droplets that are not shown, but one of the large droplets actually shrinks dramatically during one time interval. Thus we conclude that the LSW model although it correctly predicts (2), disagrees with our observations (1) and (3).

The second mechanism that causes the increase in length scale is particles or voids diffusing into contact and then coalescing. This is believed to occur in some emulsions. The droplet size distribution in emulsions has been studied and it is well described by a log-normal size distribution [27,28]. Our experimental data do not have the resolution to determine whether or not voids actually diffuse into contact; however, any mechanism whereby the voids spend long periods of time with approximately constant volumes, punctuated by events in which two adjacent voids combine relatively rapidly to form a single void, tends to yield a log-normal-like distribution. Briefly, the demonstration of this is as follows (see also Granqvist and Buhrman [29]).

Let us start at $t=0$, with a void of volume $V_{0}$. We assume that it combines in some unspecified way with another void of comparable volume. Thus after one combination event the void has a volume $2 V_{0}$. If this void also combines with a void that at this later time is again comparable in volume to itself then it has a volume $4 V_{0}$. Therefore, after $k$ combination events the volume $V_{0}$ is given by

$$
V_{k}=2^{k} V_{0} \quad \text { or } \quad \ln \frac{V_{k}}{V_{0}}=k(\ln 2) .
$$

The collisions are random events. At time $t$, the number of events is described by a Gaussian distribution $p_{t}(k)$ [30]. Thus, since the probability distribution function for $\ln V_{k}$ is Gaussian the probability distribution function for $V_{k}$ itself is log-normal. Now, we assumed, incorrectly of course, that at 
all times the voids were all of the same size. More sophisticated treatments that calculate the time evolution of $p_{t}(k)$ using Smoluchowski-type equations have been performed by Villarica et al. [31]. These calculations predicted a size distribution of the form $V^{\alpha} \exp (-V / \beta)$, i.e., a power law times an exponential decay. A distribution of this form is often called a Schulz distribution. It is different from a log-normal distribution but for $\alpha>0$ it can look rather similar to a lognormal distribution [31]. We have fitted a Schulz distribution to our data in Fig. 4. The fit is of comparable quality to that obtained with a log-normal function. Our data are not sufficient to tell whether the distribution function of the void size is log-normal or Schulz. Note that the distribution of void sizes is quite sharply peaked; the ratio between the standard deviation and the mean of the log-normal fit is 0.3 and this results in the fit parameter $\alpha=7.5$.

Our second observation was that the mean volume of a void scaled linearly with time. This is entirely consistent with a reasonable model for the rate at which voids coalesce. Perhaps the easiest way to see this is as follows. If the rate at which voids coalesce, and hence the rate of decrease of the number of voids, $n$, is proportional to the square of the number density of voids we have that $d n / d t \propto n^{2}$, for $t$ the time. This assumption that the rate is proportional to the square of the number density of voids is perhaps the most obvious assumption if the process that reduces the number of voids is a two-void process. The solution to this equation is $n \propto t^{-1}$. If the number of voids is varying as $t^{-1}$ then as the total void volume is conserved the mean void volume will increase as $t$ - the observed behavior. However, within the Smoluchowski theory [31] there is a relation between the dependence of the rate of collision of voids and their size and the rate of increase of the mean void size. In particular, our narrow distributions with $\alpha=7.5$ only result if the rate of collision of a pair of voids is a strongly decreasing function of void volume, which leads to a strongly sublinear dependence of the mean void volume on time. Thus, the theory of Villarica et al. does not describe our data quantitatively; no parameter values when input into the Smoluchowski theory will give both the correct size distribution and time dependence.

Now, in their calculations using a Smoluchowski-type theory, Villarica et al. [31] did not assume that all voids at a given time have the same size [as we did to obtain Eq. (3)]; however, they did assume that the rate at which two voids, call them $A$ and $B$, collide scales as $V_{A}^{\nu} V_{B}^{\nu}$, where $V_{A}$ and $V_{B}$, are the volumes of voids $A$ and $B$, respectively, and $\nu$ is an exponent. They also assumed that the coalescing objects were in a spatially uniform system whose properties were not changing with time. Our voids are embedded in a complex matrix that is presumably changing with time as the soluble component dissolves; thus the assumptions of spatial uniformity and time-independent collision rates may not be correct for our system. We also do not know that the collision rates are power-law functions of volume.

To summarize, the conclusions of our comparison of the data with the predictions of theory are as follows. Our observation (2) is entirely consistent with coarsening occurring via air-void coalescence, although it is also consistent with Ostwald ripening. Our observation (1), the log-normal distribu- tion, is also what we expect from coalescence, as is our observation (3) that the volumes of four large voids seem to be quite static except that the volume of one suddenly decreases, seemingly as it loses volume to the adjacent void. We conclude that coalescence via discrete events in which adjacent voids combine is consistent with all our observations, although such coalescence events cannot be observed as they occur on length and time scales that are below the resolution of our experimental techniques. Also, the predictions of an existing theory for coalescence [31] do not agree quantitatively with our data. Further experiments and theoretical calculations are needed to determine which of the assumptions made in theory are incorrect. Once we know this we will have a better understanding of the coarsening dynamics.

\section{CONCLUSIONS}

We employed the complimentary techniques of magnetic resonance imaging and $\mathrm{x}$-ray microcomputed tomography to image the air voids in a polymer-soluble-drug matrix exposed to a solvent (water). The high spatial resolution of the $\mathrm{x}$-ray $\mu \mathrm{CT}$ system allowed us to obtain the air-void size distribution as it changes over time. We observed that (1) the probability distribution function of air voids is well fitted by a log-normal distribution function, (2) the mean volume increases linearly with time, and (3) there is evidence the volume of a void can suddenly collapse, presumably as its volume is distributed to adjacent voids. All these observations are consistent with coalescence of the air voids via rapid discrete events in which one void disappears or at least shrinks rapidly while a nearby void acquires its air. Observations (1) and (3) are not compatible with the LSW mechanism of coarsening. Thus we conclude that coalescence provides a physically reasonable description of our data. We note that measuring just the time dependence of the mean void volume is not sufficient to distinguish between the LSW and coalescence models, as they both show this growth law. Therefore, an imaging technique, such as the MRI and x-ray $\mu \mathrm{CT}$, that enables the distribution function to be obtained is required. Our study is one of only a few in which this probability distribution function has been measured in situ and nondestructively.

Future work is required in order to more fully investigate the mechanism of coarsening. A good test of understanding of a future model will be whether it can adequately explain the observation that the voids form and coarsen in both regions: the region ahead of the front where water uptake is by capillary action only and the region behind the front where there is accompanying drug dissolution and therefore, presumably, greater space for particle and void movement.

Voids are unlikely to have a direct effect on the drug dissolution; motion of the air voids suggests a degree of internal mobility in the tablets which might not otherwise be possible or noticed. Moreover, there is growing evidence that in porous media, vapor diffusion through pore spaces is a significant transport mechanism [32]. It is possible that at least in the early stages of water uptake, a vapor transport mechanism and voids could facilitate wetting of the internal 
tablet surfaces. Voids are also likely to be important to structural integrity of the tablets.

The problem studied in this work is one of drug dissolution. However, the methods developed and some of the conclusions are likely to be widely applicable to water ingress into compacted powders in general, for instance, solid detergent tablet dissolution and entrapped air in hydrating cement.

\section{ACKNOWLEDGMENTS}

The authors thank Malcolm Walden and Steven Whitelock for useful discussions and help in the preparation of samples. E.K. thanks Napp Pharmaceutical Research Limited and the Engineering and Physical Sciences Research Council of the UK for financial support.
[1] C. H. Haas and J. M. Torkelson, Phys. Rev. E 55, 3191 (1997).

[2] R. Valiullin and I. Furo, Phys. Rev. E 66, 031508 (2002).

[3] S. Egelhaaf et al., Phys. Rev. E 60, 5681 (1999).

[4] P. J. Love, P. V. Coveney, and B. M. Boghosian, Phys. Rev. E 64, 021503 (2001).

[5] C. S. Brazel and N. A. Peppas, Eur. J. Pharm. Biopharm. 49, 47 (2000).

[6] I. C. Sinka, S. F. Burch, J. H. Tweed, and J. C. Cunningham, Int. J. Pharm. 271, 215 (2004).

[7] C. D. Melia, A. R. Rajabi-Siahboomi, and R. Bowtell, Pharm. Sci. Technol. Today 1, 32 (1998).

[8] S. G. Kazarian and K. L. A. Chan, Macromolecules 36, 9866 (2003).

[9] G. J. Vergote, C. Vervaet, J. P. Remon, T. Haemers, and F. Verpoort, Eur. J. Pharm. Sci. 16, 63 (2002).

[10] C. A. Fyfe, L. H. Randall, and N. E. Burlinson, J. Polym. Sci., Part A: Polym. Chem. 31, 159 (1993).

[11] L. A. Weisenberger and J. L. Koenig, Macromolecules 23, 1479 (1990).

[12] J. Siepmann, H. Kranz, R. Bodmeier, and N. A. Peppas, Pharm. Res. 16, 1748 (1999).

[13] B. J. Fahie, A. Nangia, S. K. Chora, C. A. Fyfe, H. Grondey, and A. Blazek, J. Controlled Release 51, 179 (1998).

[14] C. A. Fyfe and A. I. Blazek-Welsh, J. Controlled Release 68, 313 (2000).

[15] S. Azarmi, J. Farid, A. Nokhodchi, S. M. Bahari-Saravi, and H. Valizadeh, Int. J. Pharm. 246, 171 (2002).

[16] D. M. Omari, A. Sallam, A. Abd-Elbary, and M. El-Samaligy, Int. J. Pharm. 274, 85 (2004).
[17] M. P. Oth and A. J. Moes, Int. J. Pharm. 55, 157 (1989).

[18] P. T. Callaghan, Principles of Nuclear Magnetic Resonance Microscopy (Clarendon Press, Oxford, 1993).

[19] P. M. Jenneson, R. D. Luggar, E. J. Morton, O. Gundogdu, and U. Tuzun, J. Appl. Phys. 96, 2889 (2004).

[20] L. A. Feldkamp, L. C. Davis, and J. W. Kress, J. Opt. Soc. Am. A 1, 612 (1984).

[21] P. T. Callaghan, J. Magn. Reson. (1969-1992) 87, 304 (1990).

[22] I. M. Lifshitz and V. V. Slyozov, J. Phys. Chem. Solids 19, 35 (1961).

[23] C. Wagner, Z. Elektrochem. 65, 581 (1961).

[24] P. W. Voorhees and M. E. Glicksman, Acta Metall. 32, 2001 (1984).

[25] J. H. Yao, K. R. Elder, H. Guo, and M. Grant, Phys. Rev. B 47, 14110 (1993).

[26] E. Dickinson, C. Ritzoulis, Y. Yamamoto, and H. Logan, Colloids Surf., B 12, 139 (1999).

[27] I. Lonnqvist, B. Hakansson, B. Balinov, and O. Soderman, J. Colloid Interface Sci. 192, 66 (1997).

[28] P. J. McDonald, E. Ciampi, J. L. Keddie, M. Heidenreich, and R. Kimmich, Phys. Rev. E 59, 874 (1999).

[29] C. G. Granqvist and R. Buhrman, J. Appl. Phys. 47, 2200 (1976).

[30] M. H. DeGroot and M. J. Schervish, Probability and Statistics (Addison-Wesley, Boston, 2002).

[31] M. Villarica, M. J. Casey, J. Goodisman, and J. Chaiken, J. Chem. Phys. 98, 4610 (1993).

[32] I. Ardelean, G. Farrher, C. Mattea, and R. Kimmich, Magn. Reson. Imaging 23, 285 (2005). 